\title{
Vivencias y significados: percepciones de personas en situación de calle sobre sus diferentes momentos vitales
}

\section{Experiences and meanings: perceptions of street-people on different moments in their lives}

Paula C. Rosa ${ }^{1}$

Aceptación: 14 noviembre 2009

Aprobación: 5 mayo 2010

\section{RESUMEN}

En la actualidad en la Ciudad Autónoma de Buenos Aires miles de personas viven en plazas, veredas, calles, hogares de tránsito y paradores nocturnos. Conocer cómo viven, qué hacen durante el día, cómo llegaron a estar en esta situación y cuáles son sus expectativas futuras, es el objetivo del presente artículo. Este se centrará en las percepciones y vivencias de personas en situación de calle en torno a tres ejes de análisis: su realidad pasada, presente y futura. Estas interpretaciones fueron obtenidas a partir del trabajo en grupos focales. Los participantes de dichos grupos eran personas que pasaban sus noches en hogares de tránsito y paradores nocturnos del Gobierno de la Ciudad de Buenos Aires.

Palabras clave: Personas en situación de calle, percepciones pasadas, presentes y futuras, grupos focales.

\begin{abstract}
At present in the Autonomous City of Buenos Aires thousands of persons live in squares, paths, streets and shelters. To know how they live, what they do during the day, how they got to be in this situation and which are their future expectations it is the aim of the present article. The article will centre on the perceptions and experience of homeless people around three axes of analysis: their past, present and future reality. These interpretations were obtained from the work in focal groups. The participants of the above mentioned groups were persons who were passing their nights in shelters of the Government of the City of Buenos Aires.
\end{abstract}

Key words: Street people, past, present and future perceptions, focal groups.

Licenciada en Sociología, Doctoranda en Ciencias Sociales, Becaria doctoral de CONICET, Docente de Metodología de la Investigación Social, Facultad de Ciencias Sociales UBA (Universidad de Buenos Aires). paula_rosa00@yahoo.com.ar 


\section{Introducción}

El presente artículo se propone abordar la problemática de las personas en situación de calle a partir de la voz de sus protagonistas, es decir, hombres y mujeres de diferentes edades y trayectorias sociales que pasan o pasaron sus noches en las calles, veredas, plazas, hogares de tránsito y paradores nocturnos de la Ciudad Autónoma de Buenos Aires.

A partir del trabajo realizado en grupos focales con personas en situación de calle fue posible adentrarse en las vivencias e interpretaciones que tienen estas personas sobre su realidad pasada, presente y futura. Se buscó conocer, a partir del intercambio grupal, sus experiencias, sus estrategias, sus angustias, sus demandas, sus inquietudes y sus expectativas.

El artículo se desarrollará en función de tres ejes de análisis: la situación pasada de las personas que hoy se encuentran en situación de calle y que pasan sus noches en hogares de tránsito y paradores nocturnos del Gobierno de la Ciudad de Buenos Aires; la realidad presente: su vida cotidiana; y, por último, las propuestas y deseos para el futuro. Estos ejes se analizaron en función de los relatos individuales de los participantes y las interacciones grupales que se generaron al interior de los distintos grupos focales ${ }^{2}$.

\section{Metodología: Grupos focales}

La investigación cualitativa "se apoya sobre la idea de la unidad de la realidad, de ahí que sea holística, y en la fidelidad a la perspectiva de los actores involucrados" (Sautu, 2003). En este sentido, el enfoque fue seleccionado porque permite comprender los significados e interpretaciones que las personas que duermen en hogares y paradores del GCBA poseen de sus vivencias, "las metodologías cualitativas son apropiadas cuando el investigador se propone investigar la construcción social de significados, las perspectivas de los actores sociales, los condicionantes de la vida cotidiana o brindar una descripción detallada de la realidad" (Denzin y Lincoln, 1994).
Se consideró al grupo focal como el método cualitativo más adecuado para promover la comunicación e intercambio de experiencias con personas en situación de calle porque permite conocer cómo las personas y los grupos interpretan las vivencias por las cuales han pasado. Admite un acercamiento a la interpretación que las mismas tienen de la "realidad social" intersubjetiva. Está diseñado para dar cuenta de la manera en que los sujetos y los grupos construyen y dan sentido a los acontecimientos y circunstancias en que viven, haciendo aflorar las categorías e interpretaciones que se generan en los marcos intersubjetivos de la interacción social, por medio de procesos comunicativos y lingüísticos" (Alonso, 1998). Mediante el trabajo con grupos focales se obtienen datos, "producidos" por la interacción, sobre los conocimientos, las representaciones, las actitudes y las visiones del mundo de los participantes del mismo, "muchas veces a lo largo de este proceso de interacción grupal se amplían las opiniones, se especifican e incluso hasta se modifican, como resultado del intercambio" (Sautu, 2007).

\section{Características de los participantes:}

La convocatoria al taller fue abierta y voluntaria, es decir, que quien quería participar podía hacerlo. La invitación se hizo a los residentes de los hogares de tránsito y de paradores nocturnos que pertenecen al Gobierno de la Ciudad de Buenos Aires. Por lo cual el perfil de los participantes fue heterogéneo. Con esto hacemos referencia a que fueron hombres, mujeres y hasta niños/as (acompañados por sus madres) de diferentes edades, trayectorias sociales y lugares de nacimiento. Participaron mujeres solas jóvenes, adultas y adultas mayores y mujeres adultas con bebés y niños/as en edad escolar. Al igual que las mujeres, los hombres que participaron fueron jóvenes adultos y adultos. En su mayoría los participantes, tanto hombres como mujeres, eran oriundos de la Ciudad Autónoma de Buenos Aires y del Gran Buenos Aires; igualmente, hubo casos de participantes nacidos en alguna provincia de la República Argentina. Cabe aclarar que todos los participantes, en el momento de realizarse el taller, pasaban

Los grupos focales se llevaron a cabo en un taller participativo denominado "Dejar hablar y escuchar a las voces de los sin techo". El taller se efectuó en el marco del proyecto de investigación "Apoyo integral a los Sin Techo de la Ciudad de Buenos Aires. Ampliando el campo de lo posible” del Centro de Estudios Urbanos y Regionales CEUR/CONICET (2007-2009). 
sus noches en hogares de tránsito y paradores nocturnos. La cantidad total de participantes fue de 29 personas y fueron organizados en pequeños grupos heterogéneos en cuanto lugar de procedencia, es decir, hogar o parador. En total funcionaron tres grupos focales (contando cada uno entre 8 y 12 integrantes), cada grupo contó con un coordinador/moderador.

\section{El inicio de la situación de calle: un antes y un después}

A partir del relato de los participantes reconstruimos los motivos que los llevaron a vivir en la calle. Los puntos de inflexión en sus trayectorias de vida fueron agrupados en cuatro ejes de análisis. El primer eje fue denominado "problemas económicos", el segundo "problemas habitacionales", el tercero "problemas familiares" y el cuarto los "problemas emocionales". El armado de estos ejes permitió una profundización en el análisis de las causas que los condujeron a su situación actual.

Varios de los participantes interpretaron que llegaron a estar en esta situación de vulnerabilidad a raíz de los "problemas económicos". Entre estos podemos mencionar los generados por la pérdida del empleo. Varios de los participantes quedaron desocupados y no pudieron volver a conseguir trabajo. En otros casos, sufrieron accidentes laborales que les produjeron algún tipo de lesión física $y$, por ello, no pudieron volver a trabajar o a conseguir un nuevo empleo. Muchos de los participantes trabajaban en "negro", por lo cual no percibían ningún beneficio social; la precariedad laboral en la que trabajaban no les permitió ausentarse del trabajo para recuperarse o percibir indemnizaciones por despido o por accidentes laborales. Así nos decía Ernesto:

"[...] estuve laburando bien en la construcción, soy albañil y... después se terminó el laburo, finalización de obra, enganché en los chinos, ahí laburé 6 meses, me accidenté, [...] me resbalé, me di la boca contra la bacha y el chino no me quiso pagar, me tenía en negro [...]".

En relación a los problemas económicos se hallan los asociados a la denominada "crisis del 2001". Varios de los participantes aducen que sus problemas económicos comenzaron con el advenimiento de esta crisis política, social y económica que atravesó la Argentina a principios del siglo XXI. Consideran que la crisis fue la causa de varios de sus problemas de índole económica, así nos decía Juan: “Un tema que se planteó fue los problemas económicos del país y en lo personal, falta de dinero, falta de empleo sobre todo a partir de la crisis [...]". A causa de la falta de empleo, algunos no pudieron continuar pagando el alquiler de sus viviendas o de un cuarto de hotel o pensión, esta es la razón por la cual la pérdida de ingresos se vincula directamente con la pérdida del lugar en donde vivir. Por lo tanto, entre los motivos mencionadas por los participantes encontramos los "problemas habitacionales", es decir, la pérdida de un lugar en donde vivir, sea alquilado o bien la vivienda propia que por diferentes razones debe ser abandonada.

Otro de los motivos considerados como promotores del inicio de la vida en calle son los denominados "problemas familiares", es decir, tensiones y dificultades con los vínculos más cercanos. En muchos casos las peleas o el maltrato recibido por algún miembro de la familia los impulsó a irse del hogar. Asimismo, la separación de su pareja o la muerte de un familiar cercano fueron otras de las causas detectadas como el puntapié inicial para la vida en la calle. Una de las cuestiones que se vincula con los problemas familiares, es lo que algunos participantes enunciaron como vergüenza, muchos se sienten de este modo por lo cual no quieren acercarse a amigos y familiares para "pedir ayuda", en muchos casos estos desconocen su situación actual.

Es posible afirmar que se llega a vivir en situación de calle por la combinación de varios de los motivos mencionadas; los "problemas" se van produciendo como en cascada, uno desencadena en el otro; por ejemplo, un participante nos decía: "[...] una muerte, la de su esposa, pérdidas familiares y un accidente serio que le impide caminar normalmente". El último motivo mencionado fue el denominado: "problemas emocionales". Estos se generan a partir de la dificultad para afrontar ciertas situaciones dolorosas como la muerte de un familiar cercano. También la pérdida de 
un empleo y la dificultad de hallar otro a corto plazo fueron identificadas como causas de crisis de índole emocional Francisco describe su situación como un desencadenamiento de crisis: "crisis económica y después vino la espiritual”. Asimismo, Pablo nos decía: “[...] me quedé sin trabajo, yo hacía gas, plomería; por un hecho de la vida empecé a no querer hacer nada... después me quedé sin familia y eso me llevó a quedarme sin casa [...]".

A los problemas emocionales sumamos los relacionados con las adicciones al alcohol, a las drogas o al juego. Varios de los participantes mencionaron como desencadenante de su situación actual el problema con las adicciones. Este es el caso de Pedro, quien nos decía:

“[...] lo mío es lo más estúpido que hay, porque yo vengo de una familia muy buena, tiene, tenemos en Córdoba, Mar de Ajó en la Costa. Lo que pasa que yo me junté con un grupo de chicos que son adictos, no fumo paco, no tengo el virus...inhalo cocaína desde que tengo 15 años, fui a un centro de recuperación hace 19 años, recaí [...] no me pude levantar y no tengo el apoyo de mi familia...mi familia me abandonó, me dejó en la calle".

\section{Percepciones sobre su vida en el pasado}

Existen similitudes entre los participantes con respecto a los motivos que los llevaron a estar en situación de calle. Sin embargo, aparecieron diferencias entre ellos/as a la hora de recordar cómo fue su pasado. Así nos contaba Luis las conclusiones a las cuales habían arribado:

"[...] yo conté que había tenido una vida de cierta manera fácil en el sentido de no tener privaciones o hambre, de una familia constituida, matrimonio, hijos, hijo único, departamento en Caballito, familia de profesionales, médico, abogado, [...] puse la televisión y una mesa donde hay un plato de comida... después mis otros compañeros, bueno distintos pasados [...]".

En términos generales se podría decir que el trabajo en los grupos posibilitó distinguir entre quienes recuerdan un pasado "fe- liz" y quienes recuerdan un pasado "triste". Si bien se considera que las dicotomías como feliz-triste, bueno-malo, no son las correctas para ilustrar la diversidad y profundidad de las historias de vida, en esta experiencia fueron las que surgieron durante el intercambio de experiencias, es decir, que fue esta dicotomía la que les permitió rememorar su vida pasada. Es así que el pasado denominado como "feliz" fue asociado con una casa, con comida, con la familia, con poseer bienes materiales, con dormir en una cama, con sonrisas, con mascotas, etc. Recuerdan un pasado lleno de felicidad y comodidades. Muchos desean "volver" a ese pasado de felicidad, se podría decir que añoran ese pasado. Otros participantes recuerdan un pasado "triste", es decir, un pasado atravesado por muertes de seres queridos, enfermedades, rupturas familiares, pérdida del empleo, "malas decisiones", etc. En este sentido, varios consideran que su pasado fue la antesala de su realidad actual; aducen que las decisiones tomadas en el pasado fueron las que los condujeron a esta situación. Un participante nos decía que las causas que lo llevaron a vivir en situación de calle fueron: "no poder prever los incomodidades futuras, incapacidad para prever situaciones complicadas también, eh...y el fracaso de los emprendimientos personales [...]". Otro de los participantes nos decía: "[...] creo que indudablemente cometí errores, hubo muchas cosas que no hice, dejé de hacer y ahora cuando estoy casi cumpliendo 50".

Cabe señalar que a los participantes no les resultó fácil recordar, es decir, que fue complejo poder ahondar en su pasado, siendo este anhelado o rechazado. Las historias de vida de los participantes están marcadas por malas experiencias, rupturas, separaciones, pérdida de seres queridos, "malas decisiones", etc. Es por esto que se considera que este apartado rescata lo mejor posible, aunque escasamente, "lo que no se quiere hablar” o, mejor dicho, recordar.

\section{Su realidad presente}

A partir del relato de los participantes y del intercambio grupal podemos señalar que al pensar en su presente, sus valoraciones hicieron especial hincapié en las problemáticas que deben enfrentar día a día. Los participan- 
tes identificaron que una de las principales problemáticas con las cuales tienen que lidiar es la "cuestión laboral". Para muchos conseguir trabajo es una tarea muy difícil, consideran que el camino hacia un empleo presenta varios obstáculos. Entre estos identificaron: la falta de títulos que los habiliten, su edad avanzada, la falta de higiene corporal y/o de ropa apropiada, de un número telefónico en donde los puedan localizar y/o la falta de un domicilio fijo. Según ellos, estas cuestiones dificultan la búsqueda o la obtención de un empleo. Pedro, uno de los participantes, describe cómo las dificultades cotidianas complican su búsqueda de trabajo: "[...] uno no tiene una máquina de afeitar ¡vos no tenés con qué! Si no tenés trabajo ¿cómo haces? Te pones feo, te empezás a poner barbudo, la ropa sucia, no tenés para cambiarte de ropa, ¿quién te va a dar trabajo?".

Las personas entran en un círculo del cual es difícil salir, pues la falta de dinero les impide trasladarse a grandes distancias para aplicar a un empleo, comprar los clasificados $\mathrm{y} / \mathrm{o}$ elementos para higienizarse o tener un teléfono móvil a donde se los pueda localizar. Juan nos decía con respecto a las llamadas: "El parador es parador, permite hacer una llamada y hay que preguntar si podemos recibir llamadas, no quieren... claro...". Varios participantes nos han comentado que no pueden dar el número del parador o del hogar para que los localicen futuros empleadores. Otro de los obstáculos en la ardua búsqueda de trabajo se vincula con la falta de una dirección de referencia. Las personas no pueden dar como dirección de referencia para un trabajo la del parador u hogar. Asimismo, la falta de un domicilio impide, por ejemplo, tener una caja de ahorro. Marta, con su testimonio, agrega otra de las dificultades que genera no tener un domicilio para declarar a la hora de buscar un empleo: "Ahora yo trabajo, me van a pagar en efectivo... No quiero tener toda la plata, llevar la mochila con plata, con todo... quiero abrir una caja de ahorro, abrir una caja de ahorro, necesito un servicio a mi nombre y ¡no lo tengo!, según el banco que sea... No la puedo abrir porque no tengo un domicilio [...]".

En relación al tema del domicilio se observa una paradoja porque, por un lado, al no poder dar la dirección del parador o del hogar se les dificulta, entre otras cosas, obtener un empleo, pero, al mismo tiempo, el hecho de mencionar que viven en estos establecimientos los conduce a otro de los obstáculos que detectan los participantes, en muchos casos no han conseguido trabajo porque "viven en un hogar", así nos comentaba José: “Un muchacho [...] me dijo: "mire señor, no lo tome a mal pero haga lo siguiente: usted nunca diga cuando va a buscar un trabajo que está en un hogar porque las empresas y las casas tienen un concepto malísimo: que todos son gentes de mala vida, de la calle, ladrones, asesinos, los que mandan del Borda caen en ese lugar... Entonces haga una cosa, si tiene un teléfono particular dé un teléfono particular [...]".

Como podemos observar, el lugar en donde están viviendo les dificulta la obtención de un empleo, se podría decir que esto sucede porque quedan "pegados" a la imagen que se tiene de estos lugares y de las personas que allí viven; son lugares estrechamente asociados con la degradación social. Se tiene la concepción que estos lugares están signados por la violencia y la delincuencia, es un "espacio estigmatizado, asociado en el imaginario social con la violencia, los delitos y la anomia" (Segura, 2006), es por esto que son lugares con una imagen pública negativa. Esta imagen con la cual son asociados obstaculiza la búsqueda laboral de las personas que viven allí y contribuye a afianzar la falta de empleo entre los concurrentes porque son foco de la desconfianza y reticencia por parte de los empleadores cuando mencionan su domicilio, son blanco de "una sospecha automática de desviación [...]" (Wacquant 2007). En este sentido, Eduardo decía: “[...] desgraciadamente llevamos ese karma... es decir, nosotros en forma legal tratamos de buscar trabajo y no podemos nombrar el parador, porque es mala palabra". Se podría decir que son lugares estigmatizados que estigmatizan a quienes viven allí. Asimismo, los paradores y hogares, salvo algunos casos, se encuentran ubicados en zonas alejadas del centro de la Ciudad. Los espacios relegados en los cuales se ubican la mayoría de los establecimientos contribuyen a afianzar la concepción que se tiene de estos sitios y de las personas que allí pasan la noche. En este sentido, Nahuel nos decía: “[...] vos pensá nada más en donde están los para- 
dores, el de Retiro está en la loma del orto, en donde pasan camiones y hay una villa, el de Bepo Ghezzi también, son como lugares estratégicos a donde los ponen, en donde no pasa nadie y nadie vea nada".

Varios de los participantes mencionaron que se sienten discriminados y, en muchos casos, que se les teme. La mirada de los otros porta un juicio de valor negativo sobre su existencia. Las personas con las cuales ellos interaccionan les tienen desconfianza y hasta temor porque vivieron en la calle y/o porque viven en hogares o paradores. Las personas en situación de calle son portadoras de un estigma, tienen una marca; así nos decía Pedro: "La gente que no tiene donde vivir ino es nada! ¡No sirve para nada! Porque te dejan de lado, te dejan de lado, te corren". En este sentido Alberto nos decía: "[...] a veces la gente es ignorante, tratan mal a la gente que está sin techo... yo creo que hay que comprender, es una persona que necesita mucha ayuda, nada más que eso".

Otra de las cuestiones mencionadas como problemáticas en cuanto a la búsqueda laboral se vincula con lo que podríamos denominar la capacitación o la falta de ella. Ellos/as plantearon que necesitan estar capacitados, muchos sienten que sus conocimientos no son los suficientes como para conseguir un empleo en la actualidad. Es por esta razón que consideran que necesitan tener un título que "evidencie" sus conocimientos y habilidades. Así nos decía Marcelo: “[...] tenés que presentar el título... tu conocimiento... no te dan una pala: "agarrá una pala", no, no, te dicen: ‘ ¿a ver el título?'. En relación a la capacitación, José decía: "Si vos querés algo, capacítalo para algo así, la gente necesita un sueldo para vivir, así se paga su pieza y en ese cuadradito realiza toda su vida". Como pode- mos observar se asocia la capacitación con la posibilidad de conseguir un empleo y así dejar de vivir en un parador/hogar y comenzar una "vida", tal vez, en una pieza de hotel.

Los participantes propusieron realizar talleres de computación o de restauración de muebles o edificios. Otros contaron cómo fue su experiencia en talleres de capacitación de restauración de edificios antiguos o de fabricación de instrumentos. Así nos decía Luis: "Estoy aprendiendo un oficio, aprendiendo a hacer instrumentos, estoy en una cooperativa que se llama 'Arte sin techo' en Almagro, en donde se pretende dar algún tipo de inserción laboral". También, Alberto contó su experiencia y en su relato invita a otras personas a participar de los talleres de capacitación: "Voy a un taller, es algo que me gusta, voy a seguir adelante porque no tengo trabajo porque soy epiléptico... yo quiero decirle a la gente que no tiene lugar a donde ir que, o sea que no tiene lugar, que se acerque, yo estoy en la calle y gracias a esto estoy sobreviviendo y estoy bien $[\ldots .$.$] ".$

Horacio contaba: "En la Manzana de las Luces hay dos muchachos que comenzaron con un taller, ellos hacen guitarras, charangos, con la madera, y luego colocaron un puesto en la Boca y ahí venden los productos". En repetidas oportunidades surgió este tipo de relatos, es decir, relatos de situaciones que cambiaron gracias al hecho de haber aprendido un oficio.

\section{La vida cotidiana en paradores y hogares ${ }^{3}$}

Como se comentó anteriormente, los participantes de los grupos focales pasaban sus noches en paradores del GCBA. Una particularidad de estos establecimientos son los horarios muy estrictos de salida, es decir, una

En la Ciudad Autónoma de Buenos Aires existen diferentes programas destinados a atender a esta población. El GBCA posee sus propios hogares de tránsito y paradores nocturnos. Asimismo, subsidia establecimientos pertenecientes a organizaciones religiosas y de la sociedad civil que se ocupan de las personas en situación de calle. Entre los que pertenecen a dicho Gobierno, hay hogares/paradores para mujeres solas y mujeres con niños y para hombres solos. El GCBA posee:

Tres hogares de tránsito, uno para mujeres solas o con niños y dos para hombres solos. Los hogares ofrecen un lugar para dormir por un periodo de tiempo, alimentos, asistencia psicosocial y médica. Las personas que allí asisten conviven en el hogar debiendo compartir comidas, habitaciones, baños y tareas de limpieza.

Tres paradores nocturnos, dos para hombres solos y uno para mujeres solas o con niños. La mecánica del parador difiere del hogar de tránsito principalmente en el hecho que las personas solo pernoctan en el lugar, es decir, al otro día, una vez pasada la noche, deben retirarse. Allí reciben servicios de alimentos y de higiene como también derivaciones a hogares o consultas médicas dependiendo del caso. Si es la primera vez que asisten, tienen una entrevista con un profesional del establecimiento. 
vez terminado el desayuno deben retirarse del establecimiento. Solo pueden regresar por la tarde (alrededor de las seis de la tarde dependiendo de la estación del año), Marta comenta cuál es su situación en relación a los horarios de los paradores:

"Además otro problema de 6.30 a 9 es un tiempo que lo tenés perdido, porque para buscar trabajo las empresas abren a las 9, 10 de la mañana y hasta las 12 buscás, después no tenés a donde ir, si no llueve, perfecto, caminás, vas a una plaza, mirás vidrieras, pero si llueve no tenés lugar a donde ir, en un bar si no pagás no podes estar...[...] o sea bajo el techo conseguís un toldito hasta que pase el agua...no tenés un lugar...bueno se nos acabó lo que tenemos que hacer iy? [...]".

El testimonio de Marta pone en evidencia las dificultades que tienen durante el día si no poseen un lugar en donde estar o alguna actividad para hacer hasta el horario de regreso al parador. Otros de los participantes cuestionaron los horarios en función de sus actividades durante el día, por ejemplo, el horario de ingreso es vivido como "poco conveniente", porque si consiguen un trabajo es posible que no puedan llegar al horario estipulado de ingreso $\mathrm{y}$, por este motivo, pierdan la vacante para dormir en el establecimiento (las vacantes de los paradores se otorgan por orden de llegada, deben realizar una fila en la puerta del establecimiento hasta el horario de ingreso). Esta situación genera una gran dificultad: si consiguen un empleo que posee un horario de salida posterior al del ingreso al establecimiento, es posible que se queden sin vacante para pasar la noche y deban dormir en la calle. En este sentido Marta nos decía: "Yo conseguí un trabajo que franqueo... pero no tengo a donde ir cuando salgo ¿cuánto voy a durar así? [...] les pedí que me dejen cenar y ahí descanso un ratito... me doy una ducha y ya me voy... pero ¿cuánto tiempo?, tengo 50 años, ya no soy una pendeja... el otro día me dormí en el subte... me pasé de Congreso, eran las 6.30 y hacía 24 horas que estaba sin dormir [...]".

Como se puede observar en varios casos el planteo de los participantes se vincula con la necesidad de flexibilizar el horario para el ingreso y egreso en función de las actividades que realizan o en función de la época del año, el clima o la edad de la persona. Francisco comentaba: "Lo único que hay es una orden que los tipos dicen: "salgan a la calle"... "pero está nevando", "no importa, tienen que salir igual [...]".

Una de las cuestiones planteadas por los participantes con respecto a sus vivencias en la actualidad se vincula con la convivencia en un mismo lugar con diferentes personas y distintos modos de vivir. Una de los asuntos que los preocupa en la convivencia son los robos. Así nos decía Francisco: “[...] te roban cosas, los colgás para que se sequen y cuando volvés ya no está [...]". Otras de las cuestiones que mencionaron como una dificultad en estos lugares son las "filas". Según ellos asistir a un hogar o parador está mediado por el "hacer fila": hacen fila para entrar, para comer, para bañarse y para adquirir la toalla, los elementos de higiene o la comida; uno de los participantes nos decía: "Filas para todo, fila para esto, fila para lo otro". Muchos de los participantes, haciendo referencia a su estadía en hogares y paradores, hicieron un cuestionamiento a los coordinadores o al personal encargado de la organización de estos lugares. Varios de los participantes comentaron que recibieron "malos tratos" por parte de las personas que trabajan allí, Nahuel nos cuenta su experiencia: "Yo ahí no abro la boca ni en pedo, porque no entro más...como un tipo que es un profesional, un psiquiatra, fui, le dije que me agarré piojos, y me dijo: 'sacátelos con los dedos'. En muchos casos el trato que reciben no es el mejor y, además, consideran que es generalizado. Ariel relata su experiencia: "Encima que vas ahí, te sentís muy mal... yo en Retiro me enfermé con sarna... iyo no me agarré sarna en mi vida!... iba a estar peor que estar en la calle... no es que me queje de ellos...pero que ellos nos den una mano, yo sé que trabajan así porque les habrán hecho millones de cosas...pero todos no somos así [...]".

En este último testimonio podemos observar que Ariel reclama mayor atención por parte de las personas que trabajan en los establecimientos. También Nahuel nos decía: “[...] no me preguntan nada, no me ayudan nada [...]". Muchos reclamaron mayor contención y seguimiento por parte de los profesionales y el 
personal. En todos los relatos se evidencia la necesidad de un otro, de un otro comprensivo que se interese, que los acompañe, que los mire y que los escuche. Se podría pensar que cuando reclaman por contención y seguimiento están reclamando por una motivación, por un estímulo que les permita seguir adelante.

Más allá de las críticas realizadas a la organización de los establecimientos y al personal que allí trabaja, varios de los participantes rescataron los aspectos positivos de los hogares y paradores, y establecieron que existen diferencias en cuanto al trato y la atención entre los distintos lugares. Así nos decía Rubén: "Yo donde estoy son amables, las sábanas limpias, lo limpian todos los días, te dan shampoo, te dan jabón [...] podés pedir papel higiénico, la comida es buena, uno da las gracias que tiene donde pasar la noche y todo eso... uno agradece". También Marta nos dio su opinión: “[...] yo gracias al parador [...] estoy viva... yo no sé qué hubiera hecho [...]".

Como se puede observar no todas las experiencias son iguales y también podríamos decir que no todos los establecimientos que trabajan con personas en situación de calle se manejan del mismo modo. Sin embargo, consideramos esencial "escuchar" las demandas y necesidades que las personas exponen, como también tener en cuenta que su realidad presente, su vida cotidiana, está atravesada por obstáculos, incertidumbre, estigmatización, maltrato, precariedad y rechazo.

\section{Percepciones futuras: la "salida"}

Varios de los participantes al reflexionar sobre cómo se ven en el futuro, hicieron mención del verbo salir. Así nos decía Enrique: "Afortunadamente creo que estoy muy cerca de la salida voy a dejar esta situación". Del relato de los entrevistados se deja entrever la existencia de una frontera que divide entre el "salir" y el "entrar", el "estar afuera" y el "estar adentro". Es decir, que de esta situación en la cual se encuentran se puede "salir" o, por lo menos, se puede intentar "buscar una salida". Este es el caso de Máximo quien nos decía: "Hay que salir a buscarlos, hay que salir a la batalla, hay que salir y buscar... si se trata de dinero y bueno... buscar un trabajo [...]". En este testimonio podemos observar una alusión al movimiento: "hay que salir a buscarlos". Otros relatos hacen referencia a la idea de "estar caminando, haciendo referencia a un camino que hay que recorrer. En este sentido Luis nos decía: "Me veo caminando en un sendero hacia un lugar, espero encontrar algún tipo de solución".

La frontera divide en dos ámbitos: uno es representado como un "adentro", es el Sistema de Atención Inmediata, y el otro es el "afuera", al cual se "sale" haciendo un esfuerzo, es decir, hay que "salir a la batalla". Otro de los participantes también hacía alusión al esfuerzo, para él es necesaria una "lucha" para poder "salir", así nos decía Eduardo: "La lucha digamos... que deberíamos tener [...] es lograr una base, una organización, organizarse para salir". A partir de los testimonios podemos conocer que perciben que es necesario atravesar esta frontera para poder "salir", así nos relataba Ariel: "La idea es poder salir de este sistema, eh... estoy muy bien, pero quiero salir, poder trabajar, salir de acá y poder ver las cosas de un poquito más arriba". Varios consideran que uno de los "pasaportes de salida” es la capacitación laboral para conseguir un empleo o, bien, la obtención de un trabajo con un ingreso que les permita subsistir. Como vemos, las expectativas futuras de los participantes se vinculan con la "salida", quieren conseguir un empleo, una vivienda, una familia, una pareja, etc. Esteban aporta con su esperanza: “[...] yo creo, creer que podemos tener un futuro mejor". Si bien este apartado resulta escueto en extensión, se lo considera significativo en términos de la temática analizada. Generalmente, se hace hincapié en las cuestiones problemáticas que deben enfrentar día a día, las causas de su ingreso en la calle, el desempleo, las adicciones, etc, pero no se reflexiona sobre sus expectativas futuras o como ellos y ellas lo llaman: la "salida". Es central recalcar, y así lo cristaliza este apartado, que muchos perciben que es posible trascender su situación actual.

\section{Reflexiones finales}

El trabajo en los grupos focales fue una experiencia enriquecedora que permitió acercarse a las vivencias y significados de las personas en situación de calle en relación a los motivos que los llevaron a vivir en esta situación: un antes y un después; a conocer cómo 
es su vida cotidiana en paradores nocturnos y hogares de tránsito y, por último, a reflexionar sobre sus expectativas futuras y la tan esperada "salida". Adentrarnos en profundidad en sus interpretaciones permitió conocer cuáles son sus necesidades, cómo interpretan su situación de vulnerabilidad, cuáles son las causas y las consecuencias de la vida en calle, etc. Estas interpretaciones fueron construidas a partir del diálogo en conjunto entre todos los participantes; esto deja entrever que, en muchos casos, las vivencias son compartidas y que es posible plantear soluciones conjuntas. Conocer sus percepciones conduce a plantear soluciones afectivas acordes con sus necesidades pero a la vez permite acceder a un tipo de información que es esencial para observar los efectos de las políticas sociales y detectar los puntos que deben ser reforzados $\mathrm{y} / \mathrm{o}$ ampliados.

Estas experiencias llevan a un conocimiento profundo de determinados fenómenos sociales de notoria vigencia en la coyuntura argentina y enriquecen el propio trabajo de investigación, a partir de "escuchar" a los protagonistas.

\section{Referencias}

ALONSO, L. E. (1998), La mirada cualitativa en sociología. Una aproximación interpretativa, Madrid, Fundamentos.

DENZIN, N. y LINCOLN, Y. (1994), Handbook of Qualitative Research, Londres, Sage Publications.
DIRECCIÓN GENERAL DE ESTADÍSTICA Y CENSOS/ Ministerio de Hacienda. SEC informa: Sistema Estadístico de la Ciudad, boletín mensual, junio 2007.

ROSA, P. y GARCÍA, A. (2009), Exclusión: dilemas de una noción aplicada a situaciones de desigualdad social. En: Revista Margen de Trabajo Social y Ciencias Sociales, número 54, julio 2009.

ROSA, P. (2009), Las prácticas de encierro hoy. Reflexiones de la mano de Michel Foucault sobre los programas destinados a personas en situación de calle. Revista Afuera - Estudios de Crítica Cultural. Año IV, número 7, noviembre.

SAUTU, R. (2003), Todo es teoría. Objetivos y métodos de investigación, Buenos Aires, Ediciones Lumière.

(COMP.) (2007), Práctica de la Investigación cuantitativa y cualitativa. Articulación entre la teoría, los métodos y las técnicas, Buenos Aires, Ediciones Lumière.

SEGURA, R. (2006), Segregación residencial, fronteras urbanas y movilidad territorial. Un acercamiento etnográfico, Buenos Aires, Cuadernos del IDES N ${ }^{\circ}$ 9, Instituto de Desarrollo Económico y Social.

WACQUANT, L. (2007), Parias urbano. Marginalidad en la ciudad a comienzos del milenio, Buenos Aires, Manantial. 